Courrier du Centre International BlaisePascal

38-39 | 2017-2018

Varia

\title{
Pascal et la rhétorique biblique
}

Roland Meynet

\section{OpenEdition}

Journals

Édition électronique

URL : https://journals.openedition.org/ccibp/1340

DOI : $10.4000 /$ ccibp. 1340

ISSN : 2493-7460

\section{Éditeur}

Centre international Blaise Pascal

\section{Édition imprimée}

Date de publication : 1 janvier 2019

Pagination : 71-86

ISBN : 978-2-84516-897-8

ISSN : 0249-6674

Référence électronique

Roland Meynet, «Pascal et la rhétorique biblique », Courrier du Centre International Blaise-Pascal [En ligne], 38-39 | 2017-2018, mis en ligne le 18 juin 2021, consulté le 04 mai 2022. URL : http:// journals.openedition.org/ccibp/1340; DOI : https://doi.org/10.4000/ccibp.1340

Ce document a été généré automatiquement le 4 mai 2022.

Centre international Blaise Pascal 


\title{
Pascal et la rhétorique biblique
}

\author{
Roland Meynet
}

1 Le 27 octobre 2012, lors de la Journée d'étude internationale présidée par Philippe Sellier, et organisée par Laurent Susini dans le cadre de la Société d'Études du XVII ${ }^{e}$ Siècle, pour célébrer le trois cent cinquantième anniversaire de la mort de Pascal, j'avais été invité à exposer mon analyse du Mémorial, conduite selon les modalités de l'analyse rhétorique biblique. J'avais dû consacrer toute la première partie de mon intervention à la présentation de la rhétorique biblique, même s'il m'avait fallu le faire de manière outrageusement abrégée ${ }^{1}$. Cet exposé étant supposé connu, il est possible aujourd'hui de commencer in medias res.

2 Nos commentaires des textes bibliques se développent selon quatre rubriques : 1 . Le texte, c'est-à-dire critique textuelle, questions grammaticales et recherches lexicographiques ; 2. La composition, soit l'organisation du texte à tous ses niveaux; 3. Le contexte, historique, littéraire; 4. L'interprétation. Il va sans dire que cette dernière rubrique représente le but ultime de toutes les opérations précédentes. N'étant pas pascalien, je me limiterai ici à la seconde rubrique, la composition, laissant aux spécialistes ce qui est de leur ressort.

3 En réalité, je présenterai encore aujourd'hui la rhétorique biblique et sa technique ${ }^{2}$; cependant je ne l'illustrerai pas avec des textes bibliques, mais avec des textes tirés des Pensées. Le choix de ces textes n'est donc pas dicté par le contenu, mais par la forme, à partir du premier niveau de composition, celui du «segment" jusqu'à celui du «passage », en passant par ceux du « morceau » et de la « partie».

\section{A. Le segment}

4 Le segment peut comprendre deux membres, trois membres et même un seul membre. Le segment sera donc unimembre, bimembre ou trimembre, ou, si l'on préfère parler grec, "monostique», "distique» et "tristique». La majorité des segments sont bimembres, moins souvent trimembres, plus rarement unimembres. Cela dans la Bible ! Trop peu de textes pascaliens ont été analysés en termes d'analyse rhétorique biblique pour qu'il soit possible de se prononcer. Chacun des textes suivants est un fragment. 


\section{Segment bimembre}

Fig. 1

$\begin{array}{llll}\text { - Chacun } & \text { PEUT } & \text { mettre, } & \\ \text { - nul } & \text { NEPEUT } & \text { oter. } & \text { (S. 537) }\end{array}$

5 Les trois termes de chacun des deux membres s'opposent en parallèle.

Fig.2

\section{. Diseur de bons mots,}

- mauvais caractère. (S. 549)

6 Comme la plupart des proverbes, celui-ci est formé de deux membres qui s'opposent.

Fig.3

\section{: Voulez-vous qu'on croie dubien de vous?}

n'en dites pas.

(S. 550)

7 Les deux membres de ce proverbe sont de longueur inégale: un seul terme dans le deuxième membre au lieu de quatre dans le premier, ce qui crée un effet de surprise ${ }^{3}$.

\section{Segment trimembre}

Fig.4

+ Jésus-Christ est un Dieu

$\begin{array}{lll}\text {. dont } & \text { ON s'approche } & \text { sans orgueil } \\ \text {. et sous loquel } & \text { ON s'abaisse } & \text { sans désespoir. }\end{array}$

+ Il n'y a que deux sortes d'hommes :

- les uns justes QUISECROIENT pécheurs,

- les autres pécheurs QUISECROIENT justes.

8 Ces deux trimembres sont de type ABB'.

\section{B. Le morceau}

Que le segment soit formé d'un, de deux ou de trois membres est largement admis par les exégètes. J'ai projeté cette définition aux niveaux supérieurs, celui du «morceau » 
(formé d'un, de deux ou de trois segments), puis de la «partie » (formée d'un, de deux ou de trois morceaux).

Selon le système exposé dans le Traité de rhétorique biblique, il n'existe pas de segment « quadrimembre », ou davantage.

Fig.5

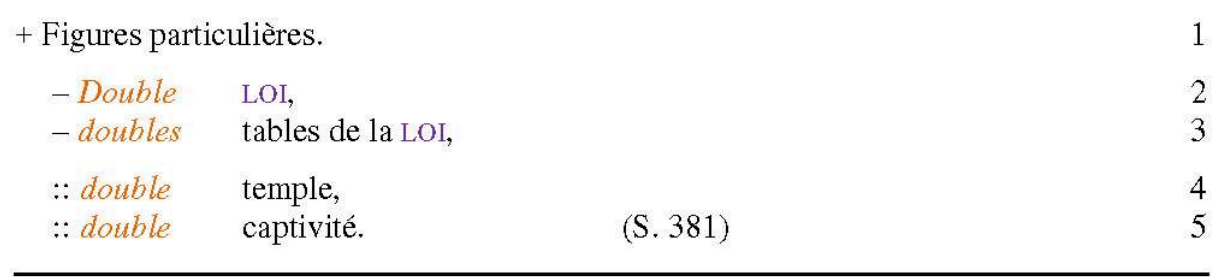

11 En effet, les membres peuvent toujours être regroupés par deux ou par trois. Ici, les deux premiers vont ensemble qui traitent de la «loi»: le premier membre fait référence au Deutéronome, le second aux deux versions du Décalogue (Ex 20 ; Dt 5). Les deux derniers membres vont ensemble, car la destruction du premier temple est liée à la captivité en Babylone comme son exorde, et le second temple ayant été construit après le retour de l'exil. Quant à la "double captivité ", il s'agit, selon la lecture des prophètes (Jr 23,7-8; déjà Os 2,16-17), de celle qu'ont souffert nos pères au pays d'Égypte puis de celle qu'ils ont connue à Babylone.

12 Avec cet exemple on passe déjà au niveau supérieur à celui du segment, c'est-à-dire à celui du «morceau » lequel est formé d'un, de deux ou de trois segments. Ici, les lignes 2-3 forment un bimembre, et de même 4-5; quant à 1 , qui est le titre du fragment, on pourra considérer que c'est un unimembre.

Le segment unimembre ne peut s'identifier qu'au niveau supérieur ${ }^{4}$.

14 Le fragment S. 381 intéresse de manière particulière le bibliste, surtout le spécialiste de rhétorique biblique, car il exprime ce que j'ai appelé la première caractéristique de la rhétorique biblique, « la binarité ${ }^{\text {». }}$

\section{Morceau formé de deux segments bimembres}

Fig.6

\begin{tabular}{llll}
\hline -ILN'EST PAS BON & & & 1 \\
$:$ d'être trop & libre. & 2 \\
-ILN'EST PAS BON & & 3 \\
$:$ d'avoir toutes & les nécessités. & (S. 90) & 4 \\
\hline
\end{tabular}

Ce fragment est de la taille d'un morceau formé de deux bimembres parallèles. Les premiers membres sont identiques, les seconds s'opposent : « être libre » signifie ne pas être soumis à quelque nécessité que ce soit, qui au contraire a «toutes les nécessités ", c'est-à-dire qui est soumis à toutes les contraintes ne saurait être libre ${ }^{6}$. 


\section{Morceau formé d'un bimembre et d'un trimembre}

Fig. 7

\begin{tabular}{|c|c|c|c|c|}
\hline $\begin{array}{l}\text { + S'il se vante } \\
+ \text { S'il s'abaisse }\end{array}$ & $\begin{array}{l}\text { je l'abaisse } \\
\text { je le vante }\end{array}$ & & & $\begin{array}{l}1 \\
2\end{array}$ \\
\hline $\begin{array}{l}:: \text { Et le contredis } \\
:: \text { Jusqu'à ce qu' } \\
=\text { Qu'il est }\end{array}$ & $\begin{array}{l}\text { toujours } \\
\text { il comprenne } \\
\text { un monstre }\end{array}$ & incompréhensible. & (S. 163) & $\begin{array}{l}3 \\
4 \\
5\end{array}$ \\
\hline
\end{tabular}

Le premier segment (1-2) est un bimembre de composition spéculaire, ou chiastique. Vient ensuite un trimembre de type AA'B (3-5). Ses deux premiers membres sont eux aussi de composition spéculaire, le troisième membre étant en quelque sorte la conclusion, ce à quoi Pascal voulait en venir ; "il comprenne » et " incompréhensible » jouent le rôle de termes finaux pour les deux derniers membres, le dernier terme faisant une chute vraiment surprenante.

Toutefois Laurent Susini suggère un autre découpage, fort tentant :

Fig.8

\begin{tabular}{|c|c|c|c|c|}
\hline $\begin{array}{l}+ \text { S'il se vante } \\
+ \text { S'il s'abaisse }\end{array}$ & $\begin{array}{l}\text { je l'abaisse } \\
\text { je le vante }\end{array}$ & & & $\begin{array}{l}1 \\
2\end{array}$ \\
\hline - Et le contredis & toujours & & & 3 \\
\hline $\begin{array}{l}:: \text { Jusqu'à ce qu' } \\
\because: \text { Qu'il est }\end{array}$ & $\begin{array}{l}\text { il comprenne } \\
\text { un monstre }\end{array}$ & incompréhensible. & (S. 163) & $\begin{array}{l}4 \\
5\end{array}$ \\
\hline
\end{tabular}

Ce seraient trois segments, deux bimembres encadrant un unimembre. Mon choix était dicté par la syntaxe, car je faisais dépendre la subordonnée de 4 (qui régit celle de 5) de la principale de 3.

19 Si au contraire on voulait faire dépendre la subordonnée de 4 des trois principales juxtaposées et coordonnées de 1-3 («je l'abaisse [...] je le vante et le contredis toujours »), il faudrait considérer 1-3 comme un trimembre de type AA’B :

Fig. 9

\begin{tabular}{|c|c|c|c|}
\hline + S'il se vante & je l'abaisse & & \\
\hline + S'il s'abaisse & je le vante & & \\
\hline + Et le contredis & toujours & & \\
\hline $\begin{array}{l}:: \text { Jusqu'à ce qu' } \\
:: \text { Qu'il est }\end{array}$ & $\begin{array}{l}\text { il comprenne } \\
\text { un monstre }\end{array}$ & incompréhensible. & (S. 163) \\
\hline
\end{tabular}

\section{Morceau formé de trois segments}


Fig. 10

\begin{tabular}{|c|c|c|}
\hline TROP et TROP PEU & de vin. & 1 \\
\hline $\begin{array}{l}\text { - Ne lui en donnez } \\
\quad:: \text { il ne peut trouver }\end{array}$ & $\begin{array}{l}\text { PAS, } \\
\text { la vérité. }\end{array}$ & $\begin{array}{l}2 \\
3\end{array}$ \\
\hline $\begin{array}{l}\text { + Donnez-Iui en } \\
\text { :: de même. }\end{array}$ & TROP, & $\begin{array}{l}4 \\
5\end{array}$ \\
\hline
\end{tabular}

L'unimembre initial est développé en deux bimembres opposés selon l'opposition entre « trop » et « trop peu », c'est-à-dire " pas » du tout. Le « de même » du dernier membre est parallèle au second membre du deuxième segment.

21 Voici un arrangement inverse - le fragment S.340, intitulé "Preuves de JésusChrist »-, où l'unimembre final fait office de conclusion :

Fig. 11

\begin{tabular}{cllr}
\hline + Jésus-Christ a dit & les choses grandes & SI SIMPLEMENT \\
.. qu'il semble & qu'il ne les a pas PENSÉES, & & 1 \\
+ et & SI nETTEMENT & néanmoins & 3 \\
.. qu'on voit bien & ce qu'il en PENSATT. & & 4 \\
CETTE CLARTÉ & jointe à CETTE NAĨVETÉ & est admirable. & 5 \\
\hline
\end{tabular}

\section{La partie}

De même que le morceau peut comprendre deux segments, trois segments et même un seul, ainsi la partie est formée d'un, de deux ou de trois morceaux.

\section{Partie formée de deux morceaux}


Fig. 12

\begin{tabular}{|c|c|c|c|}
\hline $\begin{array}{l}\text { + Qu'il serait roi } \\
\text { + et voilà ce roi }\end{array}$ & $\begin{array}{l}\text { des Juifs } \\
\text { des Juifs }\end{array}$ & $\begin{array}{l}\text { et des gentils, } \\
\text { et des gentils }\end{array}$ & $\begin{array}{l}1 \\
2\end{array}$ \\
\hline$=$ OPPRIMÉ & par les uns & et les autres & 3 \\
\hline . qui conspirent & à sa mort & & 4 \\
\hline$=$ DOMINANT & des uns & et des autres, & 5 \\
\hline$=$ et DÉTRUISANT & et le culte de Moise & dans Jérusalem, & $\begin{array}{l}6 \\
7\end{array}$ \\
\hline - dont il fait & sa première & & 8 \\
\hline$=$ & et le culte & dans Rome & 9 \\
\hline : qui en était & le centre & & 10 \\
\hline - et dont il fait & sa principale & Église. & 11 \\
\hline
\end{tabular}

\section{développé en deux trimembres qui coordonnent les deux "cultes", des Juifs et des gentils, pour former une seule "Église », unissant la « première » (8) et la " principale » (11). \\ Le fragment S. 329, intitulé «L'ordre. Contre l'objection que l'Écriture n'a pas d'ordre » est de la taille d'une partie formée de deux morceaux, le premier comprenant trois segments, le deuxième deux trimembres :}

"

Fig.13

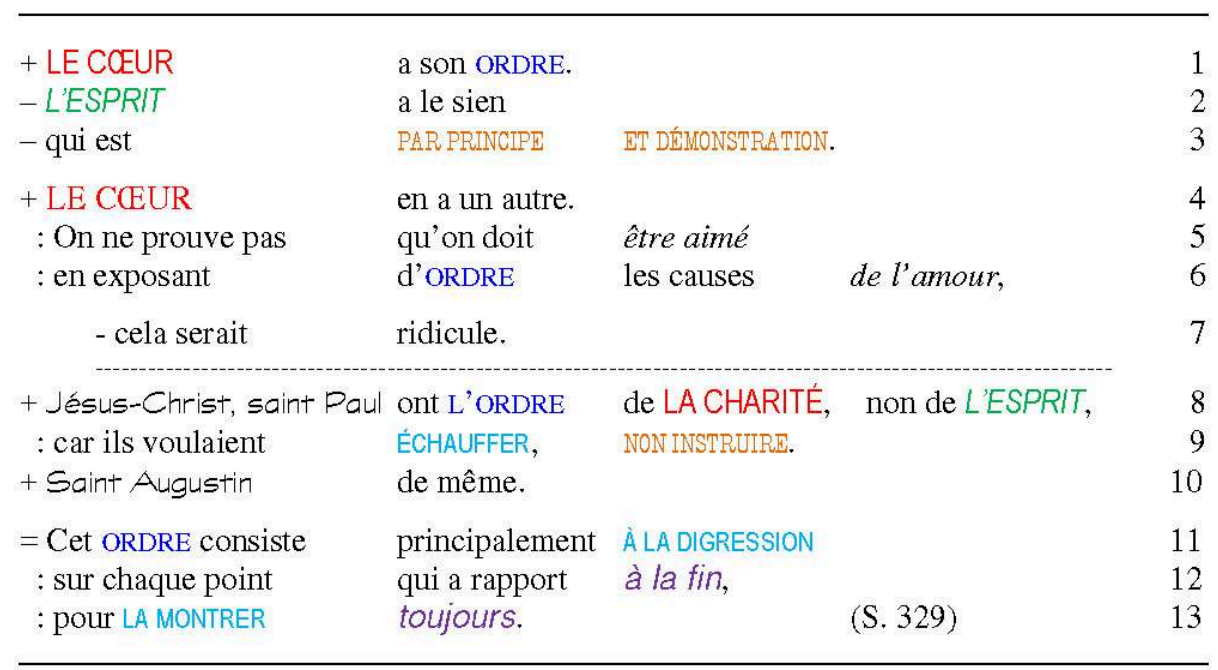

Le premier morceau (1-7) comprend deux trimembres suivis d'un unimembre. Le premier oppose l'ordre du « cœur » (1) et celui de «l'esprit » (2-3), mais seul celui de l'esprit est défini, "par principe et démonstration». Le deuxième segment semble revenir à l'ordre du « cœur » (4) mais c'est pour montrer comme il est différent de celui de l'esprit : là ne jouent pas les preuves (5) ni les « causes » (6). Et l'unimembre final (7) conclut sur le « ridicule » qu'il y aurait à projeter sur l'ordre du cœur celui de l'esprit. 
Le premier morceau disait ce que n'est pas l'ordre du cœur en l'opposant à celui de l'esprit, le deuxième morceau au contraire dira ce qu'il est. Cette fois-ci «le cœur » devient "la charité », ce qui le caractérise par rapport à la révélation chrétienne. En effet, les deux premiers termes ne sont autres que "Jésus-Christ » et «saint Paul », à quoi correspondra dans le troisième membre du premier segment "saint Augustin », le cercle s'élargissant au fil du temps. Dans le membre central de ce même segment, est énoncé l'objectif de la charité : « échauffer ", opposé à « instruire » dont on comprend que c'est le but de l'esprit. Le deuxième segment, trimembre lui aussi (11-13), va ensuite dire les moyens utilisés pour "échauffer »: une " digression » multiple et réitérée sur ce qui concerne « la fin » de l'homme, pour « montrer » "toujours » cette fin. «La fin » étant un point unique, doit être représentée « toujours » dans chacun de ses points.

« Ordre » revient deux fois dans chaque morceau $(1.6 ; 8.11)$. « Montrer » dans le second morceau (13) s'oppose à « démonstration » dans le premier (3). Il semble qu'on puisse voir aussi une opposition du même ordre entre " principe » (3) et " fin » (12).

On aura compris que le choix de ce fragment n'est pas dû au hasard pour un exposé sur " Pascal et la rhétorique biblique » : l'opposition qu'il exprime entre « démonstration » (3) et « montrer » (13) rejoint la formule par laquelle j’ai voulu synthétiser la différence entre la rhétorique classique et la rhétorique biblique: «Le Grec démontre, le Juif montre ».

Le Grec démontre, le Juif montre. La formule, forcée comme toute formule, entend souligner la différence fondamentale entre la rhétorique gréco-latine et la rhétorique biblique. Le Grec veut convaincre en imposant un raisonnement imparable, le Juif indique le chemin que le lecteur peut emprunter s'il désire comprendre ${ }^{7}$.

Voyant comment Pascal écrit, il devient naturel de le placer sur la ligne qui part de Jésus-Christ et saint Paul et va jusqu'à saint Augustin. Il faudrait voir si et comment Augustin d'Hippone abandonne la rhétorique classique de la démonstration après sa conversion pour suivre celle des textes dont il se nourrit désormais, ceux de la révélation de l'Un et de l'Autre Testament ${ }^{8}$.

Pour illustrer d'une autre manière la différence fondamentale entre la rhétorique classique gréco-romaine et la rhétorique biblico-sémitique, la mise en regard de deux prières du matin peut être éclairante ${ }^{9}$.

31 Voici la prière du matin que Jacqueline Pascal, sœur de Blaise, préconise dans son « Règlement pour les enfants », daté du 15 avril 1657 :

Dieu tout puissant,

qui nous avez fait arriver au commencement de ce jour, sauvez-nous aujourd'hui par la vertu de votre grâce,

afin que durant le cours de cette journée,

nous ne tombions dans aucun péché,

mais que dans toutes nos pensées, nos paroles et nos actions

nous n'ayons d'autre fin que d'observer vos commandements.

Ainsi soit-il ${ }^{10}$.

Bossuet a repris cette prière, dans une version un peu différente ${ }^{11}$, et elle se trouvait, sous une forme très proche, dans tous les catéchismes des diocèses de France en usage jusque dans les années 50 du siècle dernier.

Et voici maintenant la première des «bénédictions du matin » que les juifs récitent depuis des siècles : 
Béni sois-tu, Seigneur notre Dieu, roi de l'univers, qui as donné au coq l'intelligence pour discerner entre le jour et la nuit.

La prière commence avec la formule introductive de toutes les bénédictions, puis elle rappelle un verset du livre de Job :

Qui a mis dans l'ibis la sagesse

Ou qui a donné au coq l'intelligence? (Jb 38,36)

La rhétorique biblique n'est pas celle de l'exposé dogmatique qui veut convaincre l'intellect, c'est celle de la parabole qui entend toucher le cœur, pour l'inciter à réfléchir par lui-même :

c'est sans doute pourquoi les textes bibliques donnent tant à penser à l'esprit le plus exigeant, sans jamais penser à sa place. Ils propulsent leur lecteur vers le moment redoutable où il devra interpréter à son propre compte ${ }^{12}$.

\section{Partie formée de trois morceaux}

Le fragment S. 99, intitulé « Diversité » comprend trois morceaux :

Fig. 14

\begin{tabular}{|c|c|c|c|c|}
\hline $\begin{array}{l}\text { + LA THÉOLOGE } \\
\text { - mais en même } \\
\text { : combien }\end{array}$ & $\begin{array}{l}E S T \\
\text { temps } \\
\text { EST-CE }\end{array}$ & $\begin{array}{l}\text { une science, } \\
\text { de sciences? }\end{array}$ & & $\begin{array}{l}1 \\
2 \\
3\end{array}$ \\
\hline $\begin{array}{l}+\mathrm{UN} \text { HOMME } \\
- \text { mais }\end{array}$ & $\begin{array}{l}\text { EST } \\
\text { si on l'anatomise, }\end{array}$ & un suppôt, & & $\begin{array}{l}4 \\
5\end{array}$ \\
\hline $\begin{array}{l}: \text { SERA-CE } \\
: \text { les veines, } \\
: \text { le sang, }\end{array}$ & $\begin{array}{l}\text { la tête, } \\
\text { chaque veine, } \\
\text { chaque humeur }\end{array}$ & $\begin{array}{l}\text { le cœur, } \\
\text { chaque portion } \\
\text { du sang? }\end{array}$ & $\begin{array}{l}\text { l'estomac, } \\
\text { de veine, }\end{array}$ & $\begin{array}{l}6 \\
7 \\
8\end{array}$ \\
\hline $\begin{array}{l}\text { + UNE VILLE, } \\
+ \text { de loin } \\
\text { - mais à mesure qu' }\end{array}$ & $\begin{array}{l}\text { UNE CAMPAGNE, } \\
\text { C'EST } \\
\text { on s'approche, }\end{array}$ & UNE VILLE & et UNE CAMPAGNE, & $\begin{array}{r}9 \\
10 \\
11\end{array}$ \\
\hline $\begin{array}{l}: \text { CE SONT } \\
: \text { destuiles, } \\
: \text { des fourmis, }\end{array}$ & $\begin{array}{l}\text { des maisons, } \\
\text { des fouilles, } \\
\text { des jambes }\end{array}$ & $\begin{array}{l}\text { des arbres } \\
\text { des herbes, } \\
\text { de fourmis, }\end{array}$ & à l'infini. & $\begin{array}{l}12 \\
13 \\
14\end{array}$ \\
\hline+ Tout cela & s'enveloppe & sous le nom & DE CAMPAGNE. & 15 \\
\hline
\end{tabular}


campagne («c'est » : 10) s'oppose la multiplicité de leurs éléments («ce sont »:12). Le premier segment oppose "de loin» et «s'approcher ». Dans le deuxième trimembre l'énumération suit l'ordre décroissant, des «maisons" à leurs «tuiles" et des «arbres» à leurs "feuilles» et même aux «herbes" dans les deux premiers membres (12-13), pour passer ensuite aux « fourmis » et à leurs membres et finir, pour ainsi dire, "à l'infini » (14). Le segment unimembre final (15) clôt le morceau en ne considérant étrangement que « la campagne ${ }^{13}$ ».

Les trois morceaux sont parallèles : trois affirmations sont ensuite relativisées par le même "mais» (2.5.11). Bien que les morceaux soient de plus en plus développés, il semble que les deux derniers ne soient que des exemples concrets pour poser le seul problème que se pose l'auteur: les nombreuses «sciences» qui constituent «la théologie ».

\section{Le passage}

Avec le " passage », la définition change par rapport à celle des «niveaux inférieurs » que sont le « segment », « le morceau », « la partie ». Le " passage » est formé d'une ou de plusieurs parties.

40 Le fragment S. 171 est de la taille d'un passage formé de trois parties, deux plus développées aux extrémités (1-13;20-29) et une plus courte au centre (14-19).

\section{Fragment S. 171, première partie (1-13)}

41 La partie comprend trois morceaux. Le premier (1-5) est formé de deux segments qui sont autant de phrases. Le premier est un trimembre du type ABB': les deux derniers membres énumèrent ce dont sont chargés les hommes, non seulement «leur honneur ", "leur bien» et «leurs amis» (2), mais " encore » ceux de leurs amis (3), accumulation dont l'effet est accablante. Et tel est bien ce qu'explicite le bimembre suivant. Aux «affaires » de 4 , terme qui résume toutes les charges dont il a été question dans le premier segment, viennent s'ajouter "l'apprentissage des langues ", au pluriel, évidemment, et d'exercices non précisés, mais qui sont eux aussi au pluriel, ce qui concourt à souligner davantage encore la multiplicité de toutes ces charges. Les deux segments commencent par le même « on », indéterminé. 


\begin{tabular}{|c|c|c|c|c|}
\hline $\begin{array}{l}\text { + On CHARGE } \\
\text { - du soin } \\
\text { - et encore }\end{array}$ & $\begin{array}{l}\text { les hommes, } \\
\text { de leur honneur, } \\
\text { du bien }\end{array}$ & $\begin{array}{l}\text { DËs l'enfance, } \\
\text { de leur bien, } \\
\text { et de l'honneur }\end{array}$ & $\begin{array}{l}\text { DE LEURS AMIS, } \\
\text { DE LEURS AMIS. }\end{array}$ & $\begin{array}{l}1 \\
2 \\
3\end{array}$ \\
\hline $\begin{array}{l}\text { + On les accable } \\
\text { - de l'apprentissage }\end{array}$ & $\begin{array}{l}\text { D'AFFAIRES, } \\
\text { des langues }\end{array}$ & et d'exercices. & & $\begin{array}{l}4 \\
5\end{array}$ \\
\hline - Et on leur fait & entendre & & & 6 \\
\hline $\begin{array}{l}\text { : QU'ils ne sauraient } \\
\text { - sans que leur santé, } \\
\text { - et celles }\end{array}$ & $\begin{array}{l}\text { être } \\
\text { leur honneur, } \\
\text { DE LEURS AMIS }\end{array}$ & $\begin{array}{l}\text { HEUREUX } \\
\text { leur fortune } \\
\text { soient }\end{array}$ & en bon état, & $\begin{array}{l}7 \\
8 \\
9\end{array}$ \\
\hline $\begin{array}{l}\text { : et QU'une seule } \\
\text { : les rendra }\end{array}$ & $\begin{array}{l}\text { chose } \\
\text { MALHEUREUX. }\end{array}$ & qui manque & & $\begin{array}{l}10 \\
11\end{array}$ \\
\hline $\begin{array}{l}+ \text { Ainsi } \\
\therefore: \text { qui les font }\end{array}$ & $\begin{array}{l}\text { on leur donne } \\
\text { tracasser }\end{array}$ & $\begin{array}{l}\text { des CHARGES } \\
\text { DES la pointe }\end{array}$ & $\begin{array}{l}\text { et DES AFFAIRES } \\
\text { du jour. }\end{array}$ & $\begin{array}{l}12 \\
13\end{array}$ \\
\hline
\end{tabular}

Le dernier morceau (12-13) correspond au premier: il reprend le pronom " on ", «charges » et «affaires » renvoient à «charge » (1) et "affaires » (4), enfin, « dès la pointe du jour » fait inclusion avec « dès l'enfance » (1).

Le morceau central (6-11) expose la justification qu'« on » fournit aux hommes de la conduite qui leur est dictée. Le segment unimembre initial (6) est la principale. Elle est suivie de deux propositions complétives complémentaires coordonnées. Ne pas être «heureux» (7) est l'équivalent d'être « malheureux» (11). Le bonheur ne saurait être atteint si toutes les conditions énumérées dans les deux derniers membres du trimembre 7-9 n'étaient pas réunies, s'il en manquait une seule (10).

44 L'énumération du centre de la partie correspond à celle de la première (2-3). La « santé » et la « fortune » semblent expliciter « le bien » du début.

\section{Fragment S. 171, deuxième partie (14-19)}

Fig.16

\begin{tabular}{|c|c|c|c|c|}
\hline $\begin{array}{l}\text { * Voilà, } \\
\text { : de les rendre }\end{array}$ & $\begin{array}{l}\text { direz-vous, } \\
\text { HEUREUX. }\end{array}$ & une étrange & manière & $\begin{array}{l}14 \\
15\end{array}$ \\
\hline * QUE POURRAIT-ON & FAIRE & de mieux & & 16 \\
\hline : pour les rendre & MALHEUREUX? & & & 17 \\
\hline $\begin{array}{l}* * \text { Comment, } \\
\therefore: \text { CE QU'ON POURRAIT }\end{array}$ & FAIRE? & & & $\begin{array}{l}18 \\
19\end{array}$ \\
\hline
\end{tabular}

Cette courte partie comprend trois segments bimembres. On considèrera qu'ils sont organisés en deux morceaux, le premier étant ce qu'objecte l'interlocuteur supposé (14-17), le second la réaction de l'auteur (18-19). 

deux segments du premier morceau sont parallèles. Une déclaration ironique et une question rhétorique qui attend une réponse négative disent en réalité la même chose : ces gens-là ne pourraient être plus malheureux.

Fort brève, la réaction de l'auteur a la forme d'une question comme la précédente dont elle reprend les premiers termes, mais elle laisse attendre une réponse positive.

\section{Fragment S. 171, troisième partie (20-29)}

Fig. 17

\begin{tabular}{|c|c|c|c|c|}
\hline + Il ne faudrait & que leur ôter & tous CES SOINS, & & 20 \\
\hline .. car alors & ils se verraient, & & & 21 \\
\hline .. ils penseraient & à ce qu'ils sont, & & & 22 \\
\hline .. d'où ils viennent, & où ils vont. & & & 23 \\
\hline+ Et ainsi & on ne peut trop & & & 24 \\
\hline + les OCTTPER & et les détourner, & & & 25 \\
\hline - et c'est pourquoi, & après leur avoir & tant préparé & DAFFAIRES, & 26 \\
\hline - s'ils ont & quelque temps & de relâche, & & 27 \\
\hline .. on leur conseille & de l'employer & à se divertir, & à jouer & 28 \\
\hline .. et à SOCMTPER & toujours & tout entiers. & & 29 \\
\hline
\end{tabular}

Le premier morceau comprend un segment unimembre (20) suivi d'un trimembre (21-23) qui expose longuement les conséquences de ce que suggère le premier membre.

Le deuxième morceau expose ce qu'il faut faire pour «les détourner» de ces conséquences fâcheuses: "les occuper ». Les deux derniers segments envisagent la possibilité d'un « temps de relâche » (27) qui sera rempli par le divertissement (28-29). Le même « on » revient comme sujet au début des segments extrêmes.

D'un morceau à l'autre, « affaires » (26) correspond à « soins » (20); « tous » et « tout » font inclusion.

\section{Fragment S. 171, l'ensemble du passage}




\begin{tabular}{|c|c|c|c|c|}
\hline $\begin{array}{l}\text { + On CHARCE } \\
\text { - du SON } \\
\text { - et encore }\end{array}$ & $\begin{array}{l}\text { les hommes, } \\
\text { de leur honneur, } \\
\text { du bien }\end{array}$ & $\begin{array}{l}\text { dès l'enfance, } \\
\text { de leur bicn, } \\
\text { et de l'homneur }\end{array}$ & $\begin{array}{l}\text { de leurs amis, } \\
\text { de leurs amis. }\end{array}$ & $\begin{array}{l}1 \\
2 \\
3\end{array}$ \\
\hline $\begin{array}{l}+ \text { On les ACCABLE } \\
\text { de l'apprentissage }\end{array}$ & $\begin{array}{l}\text { DAFFAIRES, } \\
\text { des langues }\end{array}$ & et d'exercices. & & $\begin{array}{l}4 \\
5\end{array}$ \\
\hline $\begin{array}{l}\text { : Et on leur fait } \\
\text { : qu'ils ne sauraient }\end{array}$ & $\begin{array}{l}\text { entendre } \\
\text { être }\end{array}$ & HEUREUX & & $\begin{array}{l}6 \\
7\end{array}$ \\
\hline $\begin{array}{l}\text { - sans que leur santé, } \\
\text { - et celles }\end{array}$ & $\begin{array}{l}\text { leur honneur, } \\
\text { de leurs amis }\end{array}$ & $\begin{array}{l}\text { leur fortune } \\
\text { soient }\end{array}$ & en bon état, & $\begin{array}{l}8 \\
9\end{array}$ \\
\hline $\begin{array}{l}\text { : et qu'une seule } \\
\text { : los rerdidya. }\end{array}$ & $\begin{array}{l}\text { chose } \\
\text { HALHSUAUXY. }\end{array}$ & qui manque & & $\begin{array}{l}10 \\
11\end{array}$ \\
\hline $\begin{array}{l}+ \text { Ainsi } \\
\text { : qui les font }\end{array}$ & $\begin{array}{l}\text { on leur DONNE } \\
\text { tracasser }\end{array}$ & $\begin{array}{l}\text { des CHARGES } \\
\text { dès la pointe }\end{array}$ & $\begin{array}{l}\text { et DESAFFARES } \\
\text { du jour. }\end{array}$ & $\begin{array}{l}12 \\
13\end{array}$ \\
\hline $\begin{array}{l}\text { * Voilà, } \\
\text { = ce les rendrs }\end{array}$ & $\begin{array}{l}\text { direz-vous, } \\
\text { IEEREUX. }\end{array}$ & une étrange & manière & $\begin{array}{l}14 \\
15\end{array}$ \\
\hline $\begin{array}{l}\text { * QUE POURRAIT-on } \\
=\text { pour :os rondiro }\end{array}$ & $\begin{array}{l}\text { FAIRE } \\
\text { ZMLHEURIUXY ? }\end{array}$ & de mieux & & $\begin{array}{l}16 \\
17\end{array}$ \\
\hline $\begin{array}{l}\text { * Comment, } \\
=\text { CE QU' on POURRAT }\end{array}$ & FAIRE? & & & $\begin{array}{l}18 \\
19 \\
\end{array}$ \\
\hline - IL NE FAUDRAIT & que leur ÓTER & tous OSS SONS, & & 20 \\
\hline $\begin{array}{l}\text {.. car alors } \\
\text {. ils penseraient } \\
\text {.. d'où ils viennent, }\end{array}$ & $\begin{array}{l}\text { ils se verraient, } \\
\text { à ce qu'ils sont, } \\
\text { où ils vont. }\end{array}$ & & & $\begin{array}{l}21 \\
22 \\
23\end{array}$ \\
\hline $\begin{array}{l}\text { + Ett ainsi } \\
+ \text { + les occuper }\end{array}$ & $\begin{array}{l}\text { on ne peut trop } \\
\text { et les détourner, }\end{array}$ & & & $\begin{array}{l}24 \\
25\end{array}$ \\
\hline $\begin{array}{l}\text { - el c'est pourquoi, } \\
\text {-s'ils ont }\end{array}$ & $\begin{array}{l}\text { après leur avoir } \\
\text { quelque remps }\end{array}$ & $\begin{array}{l}\text { Lant PRÉPARÉ } \\
\text { de relâche, }\end{array}$ & DAFFARES, & $\begin{array}{l}26 \\
27\end{array}$ \\
\hline $\begin{array}{l}+ \text { on leur conseille } \\
+ \text { et à s'occuper }\end{array}$ & $\begin{array}{l}\text { de l'employer } \\
\text { toujours }\end{array}$ & $\begin{array}{l}\text { à se divertir, } \\
\text { tout entiers. }\end{array}$ & à jouer & $\begin{array}{l}28 \\
29\end{array}$ \\
\hline
\end{tabular}

51 Dans la première partie les deux occurrences de "affaires » (4.12) sont accompagnées de « soin » (2); ces termes se retrouvent dans la dernière partie (20.26). Alors que dans la première partie il s'agit de "charger » les hommes de ces affaires (1) et de les en «accabler » (4), de les leur « donner » (12), dans la dernière partie au contraire il est conseillé de leur « ôter » (20) ce qu'on leur avait pourtant « tant préparé » (26).

La partie centrale (14-19) articule les deux autres parties. "Les rendre heureux » (15) renvoie à «être heureux» dans la première partie (7); et "les rendre malheureux» (17) rappelle « les rendra malheureux» (11). Quant à « que pourrait-on faire» (16) et «ce qu'on pourrait faire » (19), ils annoncent «il ne faudrait » par quoi commence la troisième partie (20).

On remarquera que le pronom «on" revient quatre fois dans la première partie (1.4.6.12), deux fois dans la partie centrale (16.19) et de même deux fois dans la dernière partie $(24.28)^{14}$.

On remarquera surtout que se vérifie dans ce passage «la loi que la question au centre ${ }^{15} »$.

Dès le début, les fondateurs de l'analyse rhétorique, qui ont découvert les constructions concentriques, ont noté la fonction du centre de ces compositions. En 1820, Jebb appelle le centre de Mt 20, 25-28, soit les versets 26b-27: «la clé de tout le paragraphe ou strophe ${ }^{16} »$. Dans son second livre, publié en 1825, Boys écrit que le centre d'une composition concentrique joue le rôle de «clé de voûte $e^{17}$ ». En 1854, John Forbes appelle le centre « le lien» (connecting link) qui articule les autres parties du texte ${ }^{18} ;$ il précise dans une publication ultérieure : «l'idée centrale, telle un cœur, peut être le centre qui anime l'ensemble, envoyant son énergie et sa chaleur vitale jusqu'aux extrémités ${ }^{19} \%$. Comme Boys, il note que les extrémités d'une unité sont souvent en relation directe avec le centre. En 1942, soit plus d'un siècle après Jebb et Boys, Lund développera leur intuition, en fournissant un grand 
nombre d'exemples. Selon cet auteur, « le centre marque toujours le tournant [du texte] ». Telle est la première de ses sept « lois des structures chiastiques ${ }^{20}$ » (Traité, 417).

\section{Envoi}

xemples qui ont été présentés ont été soigneusement choisis pour les besoins de l'exposé. Il ne s'ensuit pas nécessairement que tous les fragments soient aussi bien composés que ceux-là. Il reste bien du travail pour voir dans quelle mesure ce genre d'analyse peut fonctionner. Nous n'en sommes encore qu'au début.

57 Il faudra évidemment pousser au-delà du niveau du « passage »; je l'ai déjà fait pour le fragment des trois ordres (S. 339) qui est de la taille d'une séquence formée de cinq passages. Un certain nombre de fragments sont de cette mesure, et même plus développés. Vaste chantier, où les procédures de l'analyse rhétorique biblique pourraient sans doute rendre quelque service.

Serait-il imaginable de pousser la recherche au-delà du fragment, pour voir si certains fragments sont organisés en ensembles plus larges? Les collages opérés par Pascal représenteraient-ils déjà une première étape dans la composition de l'Apologie qu'il avait en vue? Il est certes permis d'en douter, mais il n'est pas interdit de tenter quelque sondage. C'est aux pascaliens de dire jusqu'où il est raisonnable d'aller.

Ce sera surtout à eux, et spécialement aux plus jeunes, d'acquérir la compétence technique nécessaire pour conduire des analyses de textes de manière rigoureuse, selon la méthodologie élaborée dans le champ de l'exégèse biblique. Cette méthodologie a fait ses preuves, et non seulement sur les textes bibliques ${ }^{21}$, mais aussi sur d'autres textes de l'aire sémitique, en particulier de l'islam ${ }^{22}$. Débordant les limites géographiques du monde sémitique, d'autres auteurs pétris, comme Pascal, de Bible, ont composé eux aussi à la manière biblique, sans même s'en rendre compte : tel est le cas de la règle de saint Benoît ${ }^{23}$.

60 L'analyse rhétorique biblique est un métier. Or un métier ne s'acquiert pas d'abord par la connaissance de la théorie, même si celle-ci est indispensable. La lecture et l'étude du Traité de rhétorique biblique, ne saurait dispenser des exercices par lesquels le métier seul peut entrer. Un traité est une grammaire et chacun sait que ce n'est pas l'étude d'une grammaire qui permet d'acquérir la maitrise orale et écrite d'une langue; il y faut la pratique. Personne ne saurait faire l'économie des Exercices d'analyse rhétorique biblique $^{24}$. En ce domaine comme en d'autres, il y faut un vrai professionnalisme: l'amateurisme, comme en menuiserie ou en métallerie, est certes fort sympathique, mais ce ne sera jamais que du bricolage qui ne saurait satisfaire les exigences ni de l'esprit ni de la sagesse. 


\section{NOTES}

1. "Le Mémorial à la lumière de la rhétorique biblique ", XVII siècle, $\mathrm{n}^{\circ} 65,2013$, p. 601-619.

2. Voir R. meynet, Traité de rhétorique biblique, Paris, Lethielleux, "Rhétorique Sémitique ", $\mathrm{n}^{\circ}$ 4, 2007 (deuxième édition revue et corrigée, "Rhétorique Sémitique ", $\mathrm{n}^{\circ}$ 11, Pendé, Gabalda, 2013).

3. Autres fragments de la taille d'un segment bimembre : S. 16.77.

4. Voir, par exemple, l'analyse du Mémorial, ligne 1 dans la première partie, lignes 7 dans la deuxième (le premier morceau ne comprend qu'un seul segment unimembre).

5. Voir Traité, 15-21; sur la fonction de la binarité voir R. Meynet, Le fait synoptique reconsidéré, "Retorica Biblica e Semitica ", $\mathrm{n}^{\circ}$ 7, «Premier excursus : les deux versions du Décalogue », p. 66-100; «Deuxième excursus : les deux versions du Notre Père », p. 159-179.

6. Autre fragment formé de deux bimembres : S. 18.40.41.

7. R. Meynet, Lire la Bible, Paris, Flammarion, «Dominos», $\mathrm{n}^{\circ} 92$, 1996, p. 80 (Champs 537, Paris, Flammarion, 2003, 91) ; voir aussi Traité, 26.

8. Voir Ph. sellier, "Rhétorique et apologie : "Dieu parle bien de Dieu" ", dans id., PortRoyal et la littérature. Pascal, deuxième édition, Paris, Honoré Champion, "Champion classiques. Essais », 2010, p. 239-250.

9. Je reprends cela de mon article : « La rhétorique biblique, ou l'invitation à assumer la précarité » (www.academia.edu).

10. J. Pascal, « Règlement pour les enfants » 15 avril 1657 (à son directeur, M. Singlin), dans V. cousin, Euvres de Victor Cousin, IV eérie - Littérature, t. II, Paris, 1849, p. 241.

11. Voir Catéchisme du diocèse de Meaux publié en 1687 par J. B. Bossuet (Euvres complètes de Bossuet, t. VIII, Besançon-Paris 1840, Leçon XIX, p. 27).

12. P. Beauchamp, Préface à R. Meynet, L'Analyse rhétorique. Une nouvelle méthode pour comprendre la Bible. Textes fondateurs et exposé systématique, Paris, Éditions du Cerf, «Initiations », 1989, p. 11-12.

13. C'est un bel exemple de phénomène de clôture; voir R. Meynet, «Phénomènes de clôture dans les textes bibliques ", Annales de l'Institut de Lettres Orientales (In Memoriam Professeur Louis Pouzet), n 9, 2002-2010, p. 157-177.

14. Ce « on » omniprésent et impersonnel ressemble à s'y méprendre au «surmoi » de la psychanalyse.

15. Voir Traité, Chap. $8:$ « Le centre des compositions concentriques», A. « La question au centre », p. 417-435.

16. Sacred Literature, p. 227.

17. A Key to the Book of the Psalms, p. 123.

18. The Symmetrical Structure of Scripture, 19.76.166.173.

19. J. Forbes, Analytical Commentary on the Epistle to the Romans, p. 82. 
20. N.W. Lund, Chiasmus in the New Testament, Chapel Hill, The University of North Carolina Press, p. 40.

21. Voir le site de la «Société internationale pour l'étude de la Rhétorique Biblique et Sémitique » : www.retoricabiblicaesemitica.org.

22. Voir R. Meynet, L. Pouzet, N. Farouki, A. Sinno, Rhétorique sémitique. Textes de la Bible et de la Tradition musulmane, Paris, Les Éditions du Cerf, «Patrimoines. Religions du Livre ", 1998 (première édition arabe, 1993). M. cuypers, Le Festin. Une lecture de la sourate Al-Mâ'ida, Paris, Lethielleux, «Rhétorique Sémitique », n 3,2007 (deuxième édition, Leuven, «Rhetorica Biblica et Semitica », $n^{\circ} 10$, Peeters, 2017 ; traductions anglaise et arabe); Id., La Composition du Coran. Nazm al-Qur'ân, Paris, Gabalda, "Rhétorique Sémitique », $\mathrm{n}^{\circ}$ 9, 2012 ; Id., Une apocalypse coranique. Une lecture des trentetrois dernières sourates du Coran, Pendé, Gabalda, "Rhétorique Sémitique ", $\mathrm{n}^{\circ}$ 15, 2014.

23. Voir les contributions de Gérard Joyau dans les Actes des colloques de la RBS, édités par R. Meynet et J. Oniszczuk: http://www.retoricabiblicaesemitica.org/ retorica_biblica_fr.php ; $\quad$ http://www.retoricabiblicaesemitica.org/ retorica_biblica_semitica_fr.php.

24. R. Meynet, J. Oniszczuk, Exercices d'analyse rhétorique biblique, Pendé, Gabalda, «Rhétorique Sémitique », $\mathrm{n}^{\circ}$ 12, 2013.

\section{INDEX}

Mots-clés : rhétorique biblique, Mémorial

Keywords : biblical rhetoric, Mémorial

\section{AUTEUR}

\section{ROLAND MEYNET}

Professeur de théologie biblique à l'Université pontificale grégorienne de Rome 